\title{
Intramolecular Hydrogen Abstraction Promoted by Amidyl Radicals. Evidence for Electronic Factors in the Nucleophilic Cyclization of Ambident Amides to Oxocarbenium lons
}

\author{
Angeles Mart' in,Ine's Pe'rez-Mart'́n,and Emesto Suárez* \\ Instituto de Productos Naturales y Agrobiolog'la del C.S.I.C., Carretera de La \\ Esperanza 3, 38206 La Laguna, Tenerife, Spain
}

esuarez@ipna.csic.es

Received March 10, 2005

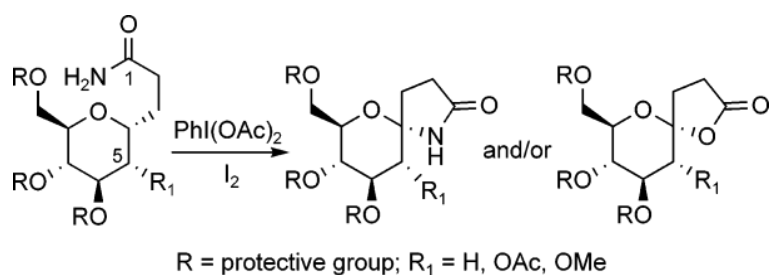

A tandem 1,5-hydrogen atom transfer/radical oxidation/nucleophilic cyclization mechanism is proposed for the intramolecular hydrogen abstraction reaction promoted for primary carboxamidyl radicals. The electron-withdrawing capacity of the $\mathrm{C}-5$ substituent can switch the reaction to give exclusively bicyclic spirolactams (6-oxa-1-azaspiro[4.5]decan-2-one) when $\mathbf{R}_{\mathbf{1}}$ ) Hor spirolactones (1,6-dioxaspiro[4.5]decan-2-one) when $\mathbf{R}_{\mathbf{1}}$ ) OAc. With a substituent of medium polarity $\left(R_{1}\right) \quad$ OMe), a mixture of lactones and lactams is formed.

Recently, the chemistry of carbon-centered radicals has driven a good deal of research due to their wide range of organic synthetic applications. ${ }^{1}$ Comparatively, heteroatomcentered radicals and nitrogen radicals in particular have received considerably less attention. ${ }^{2}$ Beginning with the pioneering studies of intramolecular hydrogen abstraction (IHA) promoted by haloamines and haloamides of Hofmann-Lo"ffler-Freytag ${ }^{3}$ and Barton, ${ }^{4}$ a number of procedures have been reported for the generation of $N$-radicals. ${ }^{1,5}$ For instance, the thermal or photochemical decomposition

(1) For reviews, see: (a) Radicals in Organic Synthesis; Renaud, P., Sibi, M. P., Eds.; Wiley-VCH: Weinheim, 2001. (b) Curran, D. P.; Porter, N. A.; Giese, B. Stereochemistry of Radical Reactions; VCH: Weinheim, 1996. (c) Gansauer, A.; Bluhm, H. Chem. ReV. 2000, 100, 2771-2788. (d) Snider, B. B. Chem. ReV. 1996, 96, 339-363. (e) Iqbal, J.; Bhatia, B.; Nayyar, N. K. Chem. ReV. 1994, 94, 519-564. (f) Melikyan, G. G. Synthesis 1993 833-850. (g) Jasperse, C. P.; Curran, D. P.; Fevig, T. L. Chem. ReV. 1991, 91,1237-1286. (h) Curran, D. P. Synthesis 1988, 417-439 and 489-513. (i) Giese, B. Radicals in Organic Synthesis: Formation of Carbon-Carbon Bonds; Pergamon: Oxford, 1986. of haloamides and the fragmentation of pyridine-2-thionoxycarbonyl amides are representative examples for the preparation of amidyl radicals.

The thermal or photolytic homolysis of $\mathrm{N}$-iodocarboxamides formed by in situ oxidation of primary amides in the presence of iodine results in the formation of $y$-lactones through a 1,5-hydrogen abstraction reaction. Despite the central interest of $\gamma$-lactones in synthetic natural products

(2) For recent reviews of the chemistry of nitrogen-centered radicals, see: (a) Stella, L. In Radicals in Organic Synthesis; Renaud, P.; Sibi, M. P., Eds.; Wiley-VCH: Weinheim, 2001; Vol. 2, pp 407-426. (b) Fallis, A. G.; Brinza, I. M. Tetrahedron 1997, 53, 17543-17594. (c) Zard, S. Z. Synlett 1996, 1148-1155. (d) Majetich, G.; Wheless, K. Tetrahedron 1995, 51, 7095-7129. (e) Esker, J. L.; Newcomb, M. In AdVances in Heterocyclic Chemistry; Katritzky, A. R., Ed.; Academic Press: New York, 1993; Vol. 58, pp 1-45. (f) Stella, L. Angew. Chem., Int. Ed. Engl. 1993, 32, 337 350. For previous reviews, see: (g) Neale, R. S. Synthesis 1971, 1-15. (h) Mackiewicz, P.; Furstoss, R. Tetrahedron 1978, 34, 3241-3260. For a recent review on the synthesis of heterocycles by radical cyclization, see: Bowman, W. R.; Bridge, C. F.; Brookes, P. J. Chem. Soc., Perkin Trans. 1 2000, 1-14. 
chemistry, this reaction has received limited attention over the years due either to low chemical yields and poor reproducibility or to the narrow scope and generality of this methodology. ${ }^{6}$ In all of the cases studied, the reaction afforded exclusively $\gamma$-lactones, with the exception of a few examples where the hydrogen to be removed was bonded to an oxygen-substituted carbon, which led to lactams instead.?

Our interest in the IHA promoted by $N$-radicals prompted us to investigate the mechanism of the apparently anomalous behavior of these amidyl radicals. ${ }^{8} \mathrm{~A}$ possible mechanism, which accounts for both $\mathrm{O}$-and $\mathrm{N}$-cyclization products, is outlined in Scheme 1 . The $C$-radical II initially formed by

Scheme 1. Tandem IHA/Radical Oxidation/Cyclization of Carboxamides

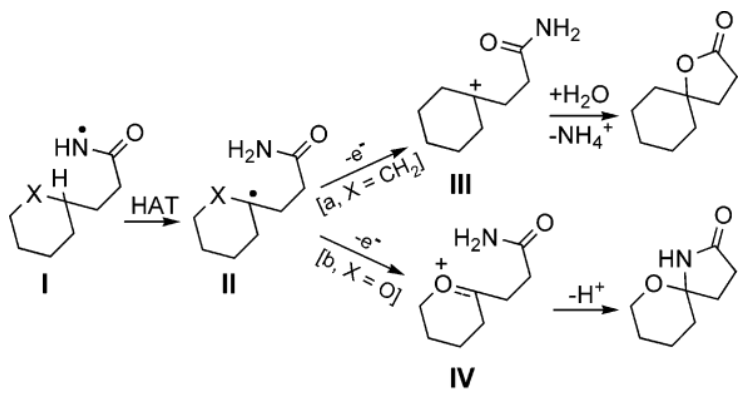

HAT = Hydrogen atom transfer

1,5-hydrogen atom transfer to the electrophilic $N$-radical I can be subsequently oxidized by the excess reagent present in the reaction medium to a carbenium ion III (path a, X) $\mathrm{CH}_{2}$ ) or an oxocarbenium ion IV (path b, X ) O). In both cases, the cyclization step proceeds through an ionic

(3)(a) Hofmann, A. W. Ber. 1883, 16, 558-560. (b) Lo"ffler, K.; Freytag, C. Ber. 1909, 42, 3427-3431.

(4) (a) Barton, D. H. R.; Beckwith, A. L. J.; Goosen, A. J. Chem. Soc. 1965, 181-190. (b) Baldwin, J. E.; Barton, D. H. R.; Dainis, I.; Pereira, J. L. C. J. Chem. Soc. 1968, 2283-2289. (c) Carruthers, W. Some Modern Methods of Organic Synthesis, 2nd ed.; Cambridge University Press: Cambridge, 1978; pp 255-257.

(5) (a) Gagosz, F.; Moutrille, C.; Zard, S. Z. Org. Lett. 2002, 4, 2707 2709. (b) Lin, X.; Stien, D.; Weinreb, S. M. Tetrahedron Lett. 2000, 41, 2333-2337. (c) Clark, A. J.; Filik, R. P.; Peacock, J. L.; Thomas, G. H. Synlett 1999, 441-443. (d) Clark, A. J.; Deeth, R. J.; Samuel, C. J.; Wongtap, H. Synlett 1999, 444-446. (e) Esker, J. L.; Newcomb, M. J. Org. Chem. 1994, 59, 9, 2779-2786. (f) Esker, J. L.; Newcomb, M. Tetrahedron Lett. 1993, 34, 6877-6880. (g) Esker, J. L.; Newcomb, M. J. Org. Chem. 1993, 58, 4933-4940.

(6) (a) Beckwith, A. L. J.; Goodrich, J. E. Aust. J. Chem. 1965, 18, 747 757. (b) Ghisalbe, E. L.; Jefferies, P. R.; Mincham, W. A. Tetrahedron 1967, 23, 4463-4468. (c) Mori, K.; Matsui, M.; Fujisawa, N. Tetrahedron 1968, 24, 3113-3125. (d) Cross, B. E.; Gatfield, I. L. J. Chem. Soc. 1971, 1539-1541. (e) Allen, J.; Bord, R. B.; McGhie, J. F.; Barton, D. H. R. J. Chem. Soc., Perkin Trans. 1 1972, 2994-3001. (f) Cross, B. E.; Firth, M. R. J. Chem. Soc., Perkin Trans. 1 1981, 3158-3160. (g) Hashimoto, Y.; Ishizone, H.; Suganuma, M.; Ogura, M.; Nakatsu, K.; Yoshioka, H Phytochemistry 1983, 22, 259-264. (h) Huneck, S.; Schmidt, J.; Porzel, A. Z. Naturforsch B 1994, 49, 561-568. For a variant of the Barton reaction in the pagodane framework, see: (i) Pinkos, R.; Rihs, G.; Prinzbach, H. Angew. Chem., Int. Ed. Engl. 1989, 28, 303-305.

(7) Herna'ndez, R.; Medina, M. C.; Salazar, J. A.; Sua'rez, E. Tetrahedron Lett. 1987, 28, 2533-2536.

(8) (a) Francisco, C. G.; Herrera, A. J.; Sua'rez, E. J. Org. Chem. 2002, 67, 1012-1017. (b) Freire, R.; Mart'in, A.; Pe'rez-Mart'in, I.; Sua'rez, E Tetrahedron Lett. 2002, 43, 5113-5116. (c) Francisco, C. G.; Herrera, A. J.; Sua'rez, E. Tetrahedron: Asymmetry 2000, 11, 3879-3882. reaction pathway that can be understood on the basis of the hard and soft acids and bases principle (HSAB). ${ }^{9}$ The oxygen atom, considered a hard nucleophile, should preferentially attack the hard electrophilic carbenium ion to give an intermediate imine which is finally hydrolyzed to the spirolactone. The softer oxocarbenium ion should preferentially attack the nitrogen atom to afford the spirolactam. ${ }^{10}$

To shed some light on this mechanism, a number of IHA experiments have been carried out. We elected to study carbohydrate-derived substrates in which the polarity of the nitrogen and the hydrogen to be abstracted could be modified by changing the substituents in the immediate neighborhood.

The nononamides outlined in Table $1 \mathbf{1}, \mathbf{4}, \mathbf{7}, \mathbf{8}, \mathbf{1 0}$, and 12 were prepared through a sequence involving (a) Lewis acid-mediated $C$-glycosidation of the corresponding 1-Oacetylcarbohydrates with allyltrimethylsilane to afford the non-8-enitols, in general with high stereoselectivity, ${ }^{11}$ (b) olefin ozonolysis followed by reductive $\left(\mathrm{NaBH}_{4}\right)$ opening of the ozonide to give the octitols, (c) transformation of the primary unprotected alcohols into the corresponding nitriles

by iodination with the $\mathrm{I}_{2} / \mathrm{Ph}_{3} \mathrm{P}$ system and subsequent nucleophilic substitution with cyanide ion, ${ }^{12}$ and (d) hydrolysis of the nitriles with $\mathrm{NH}_{4} \mathrm{OH} / \mathrm{H}_{2} \mathrm{O}_{2} \cdot{ }^{13} \mathrm{~A}$ similar sequence of reactions was used to prepare the octonamide $\mathbf{1 5}$ from the commercially available 2-deoxy-D-erythro-pentofuranose.

The $N$-radical was generated by homolytic fragmentation of a hypothetical iodoamide formed in situ by reaction of

the amide with (diacetoxyiodo)benzene (DIB) and iodine under irradiation with two $80 \mathrm{~W}$ tungsten filament lamps at room temperature.

The IHA reaction of the manno-amide 1 (entry 1) afforded a mixture of lactone 2 and lactam 3 in a $4: 6$ ratio and excellent overall yield. A similar result was observed for the cyclization of the isomeric gluco-amide 4 (entry 2). The lactone $\mathbf{5}$ and the lactam $\mathbf{6}$ obtained suggest that the change of the stereochemistry at C-5 has apparently little influence on the course and stereoselectivity of the reaction. ${ }^{14}$ Only one stereoisomer was observed for compounds $\mathbf{2}, \mathbf{3}$ and $\mathbf{5}$, 6; in all cases with the heteroatom in the R-axial position, the stability of the bicycle would undoubtedly be enhanced by the anomeric effect. The stereochemistry at C-4 of the products has been established through 2D NOESY experiments. The presence of an NOE between H-3 and H-5 in the gluco series of compounds and the absence of such an

(9)(a) Pearson, R. G. Hard and Soft Acids and Bases; Dowden, Hutchinson and Ross: Stroudsville, PA, 1973. (b) Pearson, R. G. J. Am. Chem. Soc. 1963, $85,3533-3543$.

(10) As suggested by a reviewer, a mechanism where the lactone and the lactam are, respectively, the kinetic and the thermodynamic products of an equilibrium through the cyclic imidate should also be considered.

(11) (a) Luengo, J. I.; Gleason, J. G. Tetrahedron Lett. 1992, 33, 6911 6914. (b) Giannis, A.; Sandhoff, K. Tetrahedron Lett. 1985, 26, 14791482. (c) Richter, P. K.; Tomaszewski, M. J.; Miller, R. A.; Patron, A. P.; Nicolaou, K. C. J. Chem. Soc., Chem. Commun. 1994, 1151-1152.

(12) Garegg, P. J.; Samuelsson, B. J. Chem. Soc., Chem. Commun. 1979 978-980.

(13)(a) Groziak, M. P.; Chern, J.-W.; Townsend, L. B. J. Org. Chem. 1986, 51, 1065-1069. (b) Wiberg, K. J. Am. Chem. Soc. 1953, 75, 3961- 3964 (c) Payne, G. B.; Deming, P. H.; Williams, P. H. J. Org. Chem. 1961, 26, 659-663.

(14) A control experiment (lactam 6, DIB (2.5 mmol), $\mathrm{I}_{2}(1 \mathrm{mmol}), h v$, $\mathrm{rt}, 5 \mathrm{~h}$ ) indicated that the lactam is not converted into the lactone under the reaction conditions. 
Table 1. IHA and Cyclization of Carbohydrate Amides ${ }^{a}$

\begin{tabular}{|c|c|c|c|c|}
\hline entry & substrate & $\underset{\mathrm{h}}{\mathrm{time}}$ & products & $\begin{array}{c}\text { yield } \\
\%\end{array}$ \\
\hline
\end{tabular}

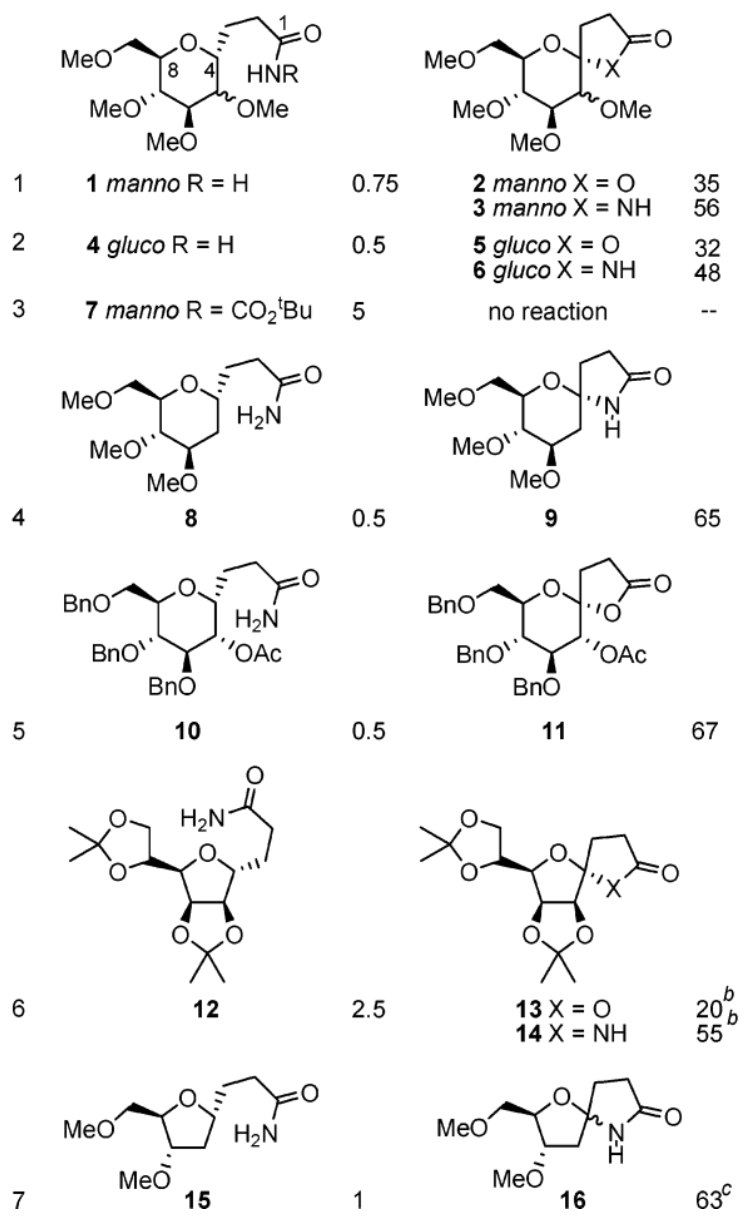

${ }^{a}$ A solution of the amide $(1 \mathrm{mmol})$ in dry $\mathrm{MeCN}(50 \mathrm{~mL})$ containing (diacetoxyiodo)benzene (DIB) $(2.5 \mathrm{mmol})$ and iodine $(1 \mathrm{mmol})$ was irradiated with two $80 \mathrm{~W}$ tungsten-filament lamps at room temperature. $b$ The $\mathrm{R} / \S$ diastereomeric ratio was $85: 15$. ${ }^{c}$ The $\mathrm{C}-4$ diastereomeric ratio was $1: 3$.

interaction in the manno series confirmed the $\mathrm{R}$-anomeric configuration. This assignment is also in agreement with the strong deshielding observed for the H-6 and H-8 in both series of compounds, due to a 1,3-diaxial interaction with the $\mathrm{R}$-heteroatom at $\mathrm{C}$-4.

It is important to mention that this is the first time that a mixture of $O$ - and $N$-cyclized compounds was obtained during the IHA promoted by amidyl radicals. This result provides the first indication that both compounds may be formed by a unique mechanism. The reaction which is, in reality, a selective oxidation of the anomeric carbon in the sugar precursor may be considered to be an intramolecular $N$ - or $O$-glycosidation.

In line with the above reasoning on the reaction mechanism, a change of the polarity of the nucleophilic amide nitrogen should promote the preferential or exclusive formation of lactones or lactams. ${ }^{15}$ Unfortunately, no hydrogen abstraction occurred when amides with a lower $\mathrm{p} K_{\mathrm{a}}$ value were used; possibly the required iodoamide and hence the $N$-radical cannot be formed under these circumstances. For example, the reaction of carbonylcarbamate 7 (entry 3) resulted in nearly complete recovery of the starting material unchanged after prolonged reaction times.

Consequently, we next turned our attention to modifying the polarity of the electrophilic oxocarbenium ion intermediate. Since a weak electron-withdrawing group (EWG) (ether) at C-5 gave a mixture of $O$ - and $N$-cyclizations (entries 1 and 2), we prepared 5-deoxy- $\mathbf{8}^{16}$ and 5-acetylcarbohydrate derivatives 10. ${ }^{17}$ This should transform the oxocarbenium ion intermediate, respectively, into a softer and harder electrophile which should have a profound influence on the cyclization with the ambident nucleophilic amide. In the first case, the reaction proceeded exclusively to give the lactam 9 through an $N$-cyclization (entry 4), which is not very surprising in light of our previous findings. ${ }^{7}$ More interestingly, the gluco amide $\mathbf{1 0}$ with an stronger EWG (ester) at C-5 afforded exclusively the lactone $\mathbf{1 1} .^{18}$

This methodology can also be extended to the synthesis of 1,6-dioxaspiro[4.4]nonan-2-one (i.e., 13) and 1-oxa-6azaspiro[4.4]nonan-7-one (i.e., 16) systems. The IHA of amide 12, prepared from 2,3:5,6-di- $O$-isopropylidene-Dmannofuranose, gave a mixture of lactone $\mathbf{1 3}$ and lactam 14 in $75 \%$ overall yield and a ratio of $25: 75$, respectively (entry $6)$. This result is similar to those described above for the synthesis of the spirobicyclic[4.5]decan-2-one systems, with an erosion in diastereoselectivity. In both compounds $\mathbf{1 3}$ and 14 the $\mathrm{R} / \S$ diastereomeric ratio was now 85:15. The 5-deoxyoctonamide $\mathbf{1 5}$ led exclusively to the expected lactam 16, isolated as a diastereoisomeric mixture at the spirocenter (ratio 1:3).

(15) For reviews on the related halocyclization of amides, see: (a) Robin, S.; Rousseau, G. Tetrahedron 1998, 54, 13681-13736. (b) Harding, K. E.; Tiner, T. H. In ComprehensiVe Organic Synthesis; Trost, B. M., Ed.; Pergamon Press: New York, 1991; Vol. 4, pp 363-421. (c) Cardillo, G.; Orena, M. Tetrahedron 1990, 46, 3321-3408. For selective lactamization of unsaturated amide derivatives, see: (d) Knapp, S.; Levorse, A. T. J. Org. Chem. 1988, 53, 4006-4014. (e) Biloski, A. J.; Wood, R. D.; Ganem, B. J. Am. Chem. Soc. 1982, 104, 3233-3235. (f) Hirama, M.; Iwashita, M.; Yamazaki, Y.; Ito, S. Tetrahedron Lett. 1984, 25, 4963-4964.

(g) Rajendra, G.; Miller, M. J. J. Org. Chem. 1987, 52, 4471-4477.

(h)Boeckman, R. K., Jr.; Connell, B. T. J. Am. Chem. Soc. 1995, 117, 12368-12369. For the use of the HSAB theory to explain the iodocyclization of unsaturated amides, see: (i) Fujita, M.; Kitagawa, O.; Suzuki, T.; Taguchi, T. J. Org. Chem. 1997, 62, 7330-7335.

(16) Synthesized from 1,3,4,6-tetra- $O$-acetyl-R-D-arabino-hexopyranose according to: (a) Beckwith, A. L. J.; Duggan, P. J. J. Chem. Soc., Perkin Trans. 2 1993, 9, 1673-1680. (b) Giese, B.; Gilges, S.; Groninger, K. S.; Lamberth, C.; Witzel, T. Liebigs Ann. Chem. 1988, 615-617. (c) Giese, B.; Groninger, K. S. Org. Synth. 1990, 69, 66-71.

(17) Synthesized from 2,6-anhydro-1,3,4-tri- $O$-benzyl-7,8,9-trideoxy-Dglycero-L-gulo-non-8-enitol according to: (a) Cipolla, L.; Lay, L.; Nicotra, F. J. Org. Chem. 1997, 62, 6678-6681. (b) Cipolla, L.; La Ferla, B.; Lay, L.; Peri, F.; Nicotra, F. Tetrahedron: Asymmetry 2000, 11, 295-303.

(18) (a) A specific signal at $V_{\max } 2270 \mathrm{~cm}^{-1}$ in the IR spectrum of the crude reaction mixture of lactone $\mathbf{1 1}$ supports the formation of ca. 6\% (as estimated by $\left.{ }^{1} \mathrm{H} \mathrm{NMR}\right)$ of the isocyanide derivative (5-O-acetyl-2,6anhydro-1,3,4-tri- $O$-benzyl-7,8-dideoxy-8-isocyanato-D- glycero-L-gulo-octitol). For the rearrangement of amides to isocyanides under analogous oxidative (lead tetraacetate) conditions, see: Baumgarten, H. E.; Smith, H. L.; Staklis, A. J. Org. Chem. 1975, 40, 3554-3561. Cross, B. E.; Firth, M. R. J. Chem. Soc., Perkin Trans. 1 1981, 3158-3160. Krecek, V.; Budesinsky, M.; Vystrcil, A. Collect. Czech. Chem. Commun. 1992, 57, 556-564. (b) The stereochemistry at the spirocenter of compound $\mathbf{1 1}$ was tentatively assigned on the basis of the observed NOESY interaction between one of the hydrogens at $\mathrm{C}-3$ and the axial $\mathrm{H}-5$. 
The somewhat lower overall yields observed in the IHA reaction of 5-deoxy-compounds $\mathbf{8}$ and $\mathbf{1 5}$ (entries 5 and 7) as compared with the reactions of amides $\mathbf{1}$ and $\mathbf{4}$ (entries 1 and 2) are probably due to a decrease in the oxocarbenium ion electrophilicity, ${ }^{19}$ which may be a factor to influence the cyclization step. On the other hand, the deactivation of the hydrogen to be abstractable for the electrophilic $N$-radical was probably responsible for the moderate yield observed in the $O$-cyclization of the amide $\mathbf{1 0}$ (entry 5) as compared with the reaction of $\mathbf{1}$ and $\mathbf{4 .}^{20}$

During the course of the present work, we became aware that the reactivity of the $\mathrm{DIB} / \mathrm{I}_{2}$ system toward the IHA of amides when the abstractable hydrogen is geminal to an unactivated carbon has never been studied. To obtain comparative data with the experiments described above, the reaction of simple cyclohexylamide derivative 17 was carried out as shown in Scheme 2. As expected and in general

Scheme 2. IHA of 3-Cyclohexylpropanamide (17) ${ }^{a}$

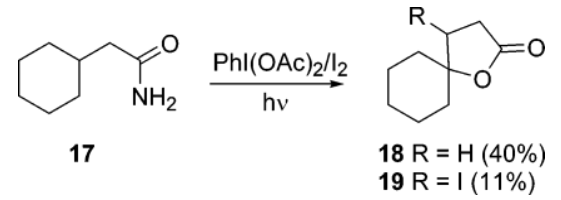

${ }^{a}$ The low yield of $\mathbf{1 8}$ and $\mathbf{1 9}$ was probably due to their high volatility.

agreement with the results obtained with other oxidizing systems, the cyclization reaction led only to lactones $\mathbf{1 8}$ and

(19) Influence of the substituents on the reactivity of tetrahydropyran oxocarbenium ions: (a) Ayala, L.; Lucero, C. G.; Romero, J. A. C.; Tabacco, S. A.; Woerpel, K. A. J. Am. Chem. Soc. 2003, 125, 15521-15528.

(b) Smith, D. M.; Woerpel, K. A Org. Lett. 2004, 6, 2063-2066.

(20) For evidence of a deactivating influence of a \$-oxygen in the intramolecular hydrogen abstraction reaction, see: (a) Francisco, C. G.; Freire, R.; Herrera, A. J.; Pe'rez-Mart'in, I.; Sua'rez, E. Org. Lett. 2002, 4 1959-1961. In intermolecular reactions, see: (b) Busfield, W. K.; Grice, I. D.; Jenkins, I. D.; Monteiro, M. J. J. Chem. Soc., Perkin Trans. 2 1994, 1071-1077. (c) Busfield, W. K.; Grice, I. D.; Jenkins, I. D. J. Chem. Soc., Perkin Trans. 2 1994, 1079-1086.
19, no lactams being detected in the crude reaction mixture. The iodo lactone $\mathbf{1 9}$ is undoubtedly the result of an $O$ iodolactonization of a 3-cyclohexylidenepropanamide intermediate (not isolated).

In light of the results attained, it seems likely that the reaction proceeds in two principal steps involving a radicaldriven hydrogen atom transfer followed by an ionic nucleophilic cyclization. This occurs for the carbohydrate examples studied here where the $C$-radical initially formed can be oxidized to an oxocarbenium ion. Most probably, the mechanism can be extended to other systems where an intermediate carbenium ion is formed. The electrophilicity of the oxocarbenium ion can be modulated by tuning the electron-withdrawing ability of the substituent at C-5 to trigger the reaction specifically to give spirolactones or spirolactams. Indeed, the correct choice of the substituents at C-5 in 4,8-anhydro-2,3-dideoxynononamides can be the switch for either the preparation of 1,6-dioxaspiro[4.5]decan2-one or 6-oxa-1-azaspiro[4.5]decan-2-one bicyclic systems.

Analogously, the bicycles 1,6-dioxaspiro[4.4]nonan-2-one or 1-oxa-6-azaspiro[4.4]nonan-7-one can be prepared starting from monosaccharides in furanose form. This reaction is a nice example not only of the $O$ - and $N$-ambident nucleophilic reactivity of the amide group but also of the umpolung reactivity of the nitrogen during the reaction: electrophilic character during the radical hydrogen transfer and nucleophilic in the ionic cyclization step.

Acknowledgment. This work was supported by the Investigation Program Nos. CTQ2004-06381/BQU and CTQ2004-02367/BQU of the Ministerio de Educacio'n y Ciencia, Spain, cofinanced with the Fondo Europeo de Desarrollo Regional (FEDER). I.P.-M. thanks the Program I3P-CSIC for a fellowship.

Supporting Information Available: Detailed experimental procedures and spectral and analytical data for compounds 1-16, 19, and precursors. This material is available free of charge via the Internet at http://pubs.acs.org. 